\title{
NEUTROPENIA FEBRIL EM PACIENTES COM CÂNCER DE MAMA SUBMETIDAS À QUIMIOTERAPIA: EXPERIÊNCIA DE 12 ANOS
}

\author{
Omero Benedicto Poli Neto, Francisco José Candido dos Reis*, Fernando Muniz Lopes, \\ Hélio Humberto Angotti Carrara, Jurandyr Moreira de Andrade \\ Trabalho realizado no Hospital das Clínicas da Faculdade de Medicina \\ de Ribeirão Preto da Universidade de São Paulo, Ribeirão Preto, SP
}

RESUMO - OBJeTIVOs. Identificar as características das pacientes com câncer de mama que desenvolveram neutropenia febril, estabelecer fatores de risco para a sua ocorrência e indicadores de evolução desfavorável.

Métodos. Realizamos um estudo caso-controle com 65 pacientes. Foram incluídas 13 pacientes que desenvolveram neutropenia febril e quatro controles por caso pareados por data e número de ciclos de quimioterapia prévios, drogas e doses empregadas. Os dados clínicos e laboratoriais foram obtidos dos prontuários médicos. Utilizamos odds ratio (OR) e intervalo de confiança (IC) de $95 \%$ para estimar a significância dos fatores de risco.

Resultados. Identificamos dois fatores de risco associados à ocorrência de neutropenia febril: a realização de quimioterapia nas primeiras 24 horas após a cirurgia (OR: I59,9 IC 95\%: 9,5 a 2699) e a realização concomitante de quimioterapia e radioterapia da mama (OR: 108,3 IC 95\%: 4,9 a 239I). Não observamos diferenças significativas entre casos e controles quanto à idade, índice de massa corporal e contagem de neutrófilos e monócitos antes da quimioterapia. Três pacientes foram a óbito $(23,1 \%)$. Duas delas tinham idade superior a 60 anos, não apresentavam comorbidades, tinham recebido o primeiro ciclo de CMF nas primeiras 24 horas após a cirurgia e tiveram infecção de sítio cirúrgico.

Conclusóes. Os principais fatores de risco associados a neutropenia febril em pacientes com câncer de mama foram quimioterapia nas primeiras 24 horas após a cirurgia, e uso concomitante de quimioterapia e radioterapia da mama. Nosso estudo mostra, portanto, que estas situações devem ser evitadas.

UnITERMOS: Neutropenia febril. Câncer de mama. Quimioterapia. Radioterapia.

\section{INTRODUÇÃO}

A neutropenia febril é uma grave complicação do uso de agentes quimioterápicos em geral. Sua ocorrência associada à quimioterapia para o câncer de mama pode interferir negativamente na continuidade do tratamento e na qualidade de vida da paciente. Isso acontece em virtude de internações prolongadas, complicações, e atraso na aplicação do ciclo de quimioterapia subseqüente!

A incidência de neutropenia febril nestas pacientes é baixa quando comparada ao tratamento de outras doenças, cerca de 10\%². Entretanto, a taxa de complicações decorrentes é elevada acarretando importante morbidade e mortalidade, a taxa de óbitos varia de $4 \%$ a $21 \%{ }^{3}$. Esta condição está associada ainda a um aumento importante nos custos do manejo das pacientes com câncer de mama ${ }^{24,5}$.

\footnotetext{
* Correspondência:

Departamento de Ginecologia e Obstetrícia Av. Bandeirantes' $3900-8^{\circ}$ andar

CEP: 14049-900 - Ribeirão Preto - SP Tel: (16) 602-2589

E-mail: ficreis@fmrp.usp.br
}

A principal estratégia para a prevenção da ocorrência de neutropenia febril baseia-se na identificação de fatores de risco. Os principais fatores de risco descritos para pacientes com câncer de mama que receberam quimioterapia são: idade superior a 60 anos, regimes contendo fluoracil, reserva medular óssea comprometida pela doença de base ou pelo uso prévio de terapia mielossupressiva, e radioterapia prévia ou concomitante à quimioterapia ${ }^{6}$. Apesar da elevada incidência do câncer de mama em nosso meio, não encontramos casuísticas nacionais publicadas a respeito do assunto até o momento.

O objetivo deste estudo foi identificar as características das pacientes com câncer de mama que desenvolveram neutropenia febril após quimioterapia, e estabelecer fatores de risco para a sua ocorrência e indicadores de evolução desfavorável.

\section{Métodos}

Realizamos um estudo caso-controle no qual incluímos 65 pacientes com câncer de mama tratadas com quimioterapia no período de janeiro de 1990 a dezembro de 2002.
Foram 13 casos e 52 controles. Os casos foram todas as pacientes internadas no serviço com diagnóstico de neutropenia febril após quimioterapia no período. Utilizamos como critério de inclusão: história de febre e contagem de neutrófilos inferior a 1000 células $/ \mathrm{mm}^{3[5]}$ ou inferior a $40 \%$ do número absoluto de glóbulos brancos ${ }^{7}$. Descrevemos os resultados de culturas microbiológicas e radiografias de tórax, o sítio de infecção, o uso de filgrastime e a evolução clínica. Os antibióticos utilizados no tratamento eram padronizados pela Comissão de Controle de Infecção Hospitalar do nosso serviço e incluíram ao longo do período de estudo: ceftriaxone e amicacina, ceftazidima e amicacina, cefepime e amicacina. Vancomicina, fluconazol e outras drogas somente foram utilizadas após identificação de agente específico ou necessidade clínica: piora do quadro clínico ou ausência de resposta ao tratamento inicial por mais de 48 horas. Selecionamos quatro controles para cada caso pareados por: data da realização e número de ciclos de quimioterapia prévios, drogas e doses empregadas. 
Poli Neto OB et Al.

As variáveis analisadas foram: idade, índice de massa corporal, status menopausal, presença de comorbidades, estadio da doença segundo a UICC ${ }^{8}$, indicação da quimioterapia, uso da quimioterapia dentro das primeiras 24 horas após a cirurgia, uso de quimioterapia e radioterapia concomitante da mama (definida como sobreposição de ao menos 21 dias no tratamento concomitante), contagem de neutrófilos e monócitos pré e pós-quimioterapia. Para avaliar o risco de complicação depois de instalada a neutropenia febril utilizamos um escore proposto por Hughes et al. ${ }^{6}$, o qual contempla: sintomas da doença, hipotensão, dor abdominal, desidratação, doença pulmonar obstrutiva crônica, temperatura superior a 39 C, início da febre em ambiente hospitalar e idade superior a 60 anos. Um escore igual ou superior a 21 indica uma paciente de baixo risco para complicações e morbidade.

A análise dos dados foi realizada com auxílio dos programas GraphPad Software 3.0 e Win Episcope 2.0. Utilizamos proporção atribuível (PA) e odds ratio (OR) para estimar a significância dos fatores de risco, com intervalo de confiança (IC) de 95\%.

\section{Resultados}

As principais características das pacientes estão resumidas na Tabela I. Observamos dois fatores de risco associados à evolução para neutropenia febril: a realização de quimioterapia dentro das primeiras 24 horas após a cirurgia (OR: I 59,9 IC 95\%: 9,5 a 2699) e a realização concomitante de quimioterapia e radioterapia da mama (OR: 108,3 IC 95\%: 4,9 a 239 I) (Tabela 2). Não observamos associação entre a ocorrência de neutropenia febril e idade superior a 60 anos, índice de massa corporal superior a $25 \mathrm{Kg} / \mathrm{m}^{2}$, menopausa, presença de comorbidade ou doença em estadio avançado (IIB, III, IV).

Os casos e os controles foram semelhantes quanto à contagem de neutrófilos e monócitos antes da quimioterapia, quanto aos agentes quimioterápicos utilizados e número de ciclos prévios. Das pacientes que desenvolveram neutropenia febril, seis tinham recebido quimioterapia neoadjuvante, cinco pacientes tinham recebido quimioterapia adjuvante e duas pacientes tinham recebido quimioterapia paliativa. Apenas uma paciente tinha alto risco para complicações com escore de risco inferior a 21 .

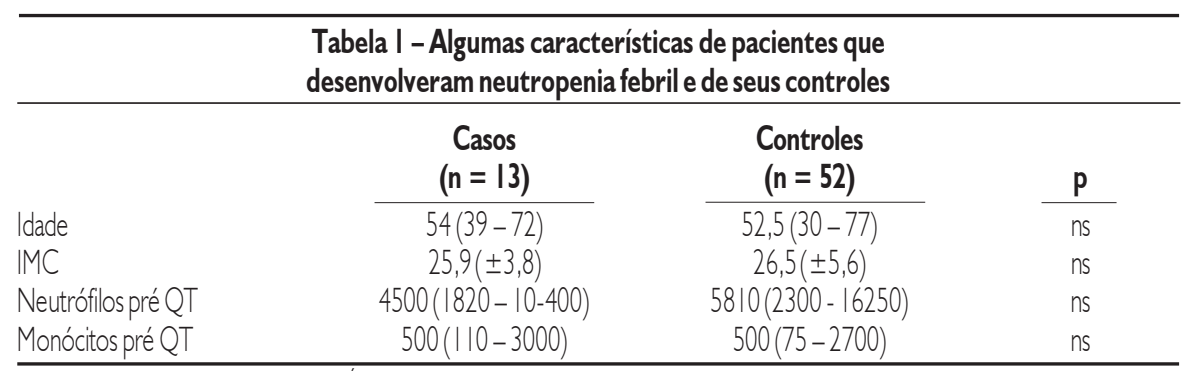

Idade em anos: mediana (intervalo); IMC (índice de massa corporal) em Kg/m²: média (desvio padrão); QT: quimioterapia; ns: não significativo (p > $0,05)$

Tabela 2 - Fatores de risco para a ocorrência de neutropenia febril em pacientes com câncer de mama que receberam quimioterapia

\begin{tabular}{|c|c|c|c|}
\hline & PA & OR & IC 95\% \\
\hline $\begin{array}{l}\text { Idade }>60 \\
\text { IMC > } 25 \\
\text { Menopausa } \\
\text { Comorbidade } \\
\text { Estadio avançado } \\
\text { PO imediato } \\
\text { RT concomitante }\end{array}$ & $\begin{array}{l}0,05 \\
- \\
0,68 \\
- \\
0,06 \\
0,23 \\
0,16\end{array}$ & $\begin{array}{c}1,2 \\
0,6 \\
5,0 \\
0,3 \\
1,1 \\
159,9 \\
108,3\end{array}$ & $\begin{array}{c}0,3-4,2 \\
0,1-2,3 \\
0,8-30,5 \\
0,1-1,8 \\
0,3-3,8 \\
9,5-2699,0 \\
4,9-2391,0\end{array}$ \\
\hline
\end{tabular}

Idade em anos. IMC (Índice de massa corporal) em Kg/m². PO imediato: paciente que recebeu quimioterapia nas primeiras 24 horas após a cirurgia. RT concomitante: paciente que recebeu quimioterapia concomitante com radioterapia da mama. OR: odds ratio. PA: proporção atribuível. IC $95 \%$ : intervalo de confiança de $95 \%$.

A mediana do tempo de internação foi cinco dias, variando de 3 a 14 dias, independente do uso ou não de filgrastime. Quatro pacientes tiveram o diagnóstico radiológico de pneumonia, sendo que três delas apresentavam queixas associadas ao comprometimento do sistema respiratório. Amostra sangüínea para cultivo microbiológioco foi colhida de todas as 12 pacientes, sendo positiva em apenas um caso (Staphylococcus aureus). Amostra de urina para cultivo microbiológico foi colhida de dez pacientes, sendo positiva em apenas um caso (Escherichia coll). Os diagnósticos mais comuns de infecção foram pneumonia: quatro casos; candidíase oral: quatro casos; infecção em sítio cirúrgico: três casos; amigdalite purulenta: dois casos; flebite: um caso com sintomas; infecção urinária: um caso com sintomas; cinco pacientes tiveram ao menos dois diagnósticos de infecção. Seis pacientes usaram filgrastime. Três pacientes foram a óbito: duas pacientes tinham idade superior a 60 anos, não apresentavam comorbidades, tinham recebido o primeiro ciclo de CMF (ciclofosfamida, metotrexate, fluoracil) nas primeiras 24 horas após a cirurgia e tiveram infecção de sítio cirúrgico. Uma delas apresentava escore de risco 19 e a outra apresentava contagem de neutrófilos de 100 células $/ \mathrm{mm}^{3}$, indicador de neutropenia severa. Ambas usaram filgrastime terapêutico. A terceira paciente havia recebido o primeiro ciclo de quimioterapia paliativa, teve o diagnóstico de pneumonia na internação e apresentava um importante quadro de confusão mental.

\section{Discussão}

No nosso estudo a ocorrência de neutropenia febril esteve associada à realização concomitante de radioterapia e à realização de quimioterapia nas primeiras 24 horas após a cirurgia. Este último também foi importante indicador de evolução desfavorável em pacientes com neutropenia febril: duas das três pacientes neutropênicas febris que realizaram quimioterapia nas primeiras 24 horas após a cirurgia foram a óbito.

O papel da cirurgia na determinação de neutropenia febril não está completamente elucidado. Alguns trabalhos têm mostrado que a manutenção das funções de neutrófilos e monócitos no pós-operatório depende de suas características antes do procedimento cirúrgico. Através da análise de marcadores fenotípicos de ativação funcional de monócitos e polimorfonucleares, Barry et al. observaram 
que pacientes submetidas a cirurgias de médio e grande porte podem apresentar comportamento imunológico variável, ora com melhora da resposta imune após o estresse cirúrgico, ora com piora. Os autores creditam esses padrões distintos de respostas de monócitos e polimorfonucleares ao grau de estresse préoperatório e fatores genéticos, uma vez que não observaram correlações com a idade, a severidade da cirurgia e o sexo do paciente ${ }^{9}$. Além disso, Ciepichal \& Kubler observaram uma importante redução da atividade fagocitária no pós-operatório que persistia por ao menos 24 horas. Após anestesias de mais de 2 horas, particularmente quando se usa halotano, há importante redução da quimiotaxia e da atividade fagocitária e bactericida dos neutrófilos ${ }^{10}$. Com os dados do nosso estudo e os dados da literatura, concluímos que a quimioterapia não deve ser realizada nas primeiras 24 horas após a cirurgia e sugerimos que se aguarde o restabelecimento das condições clínicas prévias à cirurgia.

A seqüência ótima de quimioterapia e radioterapia permanece controversa. Alguns autores têm mostrado que a realização concomitante de quimioterapia e radioterapia não interfere no tratamento da paciente " , afirmando que o regime de CMF e radioterapia concomitante é aceitável e está associado a baixo risco de toxicidade séria ${ }^{[12]}$, assim como o uso concomitante de FAC (fluoracil, doxorrubicina e ciclofosfamida) e radioterapia ${ }^{[13]}$. Apesar de chegarem à mesma conclusão, Dubey et al. observaram 16 casos de neutropenia severa em I 12 pacientes submetidas ao tratamento concomitante de CMF e radioterapia $^{14}$. Fiets et al., por sua vez, consideraram que o uso concomitante de CMF ou AC (doxorrubicina e ciclofosfamida) e radioterapia leva a níveis inaceitáveis de toxicidade aguda e deve ser evitado ${ }^{15}$. Outros autores observaram que o uso de CMF e radioterapia concomitante pode promover a necessidade de redução na dose do quimioterápico, além de culminar em menor contagem de leucócitos durante a terapia com possível atraso na terapêutica ${ }^{16,17}$. Além disso, não há evidências de prejuízo no tratamento de pacientes, desde que o atraso na realização da radioterapia não exceda 20 a 24 semanas da cirurgia ${ }^{18}$. Nossos dados mostraram que a neutropenia febril esteve associada ao uso concomitante de quimioterapia e radioterapia da mama, mas não foi um indicador de evolução desfavorável. Com base nestas observações e frente aos demais dados da literatura, sugerimos que o uso concomitante de quimioterapia e radioterapia da mama deve ser evitado.

A literatura afirma que os sinais e sintomas de inflamação podem ser mínimos ou ausentes em pacientes com neutropenia febril ${ }^{19}$. Entretanto, todas as pacientes do nosso estudo apresentaram febre. Apenas uma paciente com diagnóstico de pneumonia, não tinha sintomas associados ao sítio de infecção. Obtivemos crescimento de bactérias em apenas duas culturas de pacientes distintas: Staphilococcus aureus em hemocultura e Escherichia coli em urocultura. A identificação desses agentes coincide com a relação dos principais agentes causadores de infecção em pacientes neutropênicos febris. A IDSA (Infectious Diseases Society of America) orienta a coleta de hemograma completo e uma ou mais amostras sangüíneas para hemocultura para todas as pacientes. Entretanto, culturas de urina, reto, nasofaringe e orofaringe devem ser realizadas apenas quando sinais ou sintomas de comprometimento do sistema referido estiverem presentes. Em nosso estudo, I I pacientes foram submetidas à radiografia de tórax, sendo feitos quatro diagnósticos de pneumonia, dos quais apenas uma paciente era assintomática. Hughes et al. sugerem que a radiografia de tórax deve ser realizada apenas quando há sinais ou sintomas relacionados ao comprometimento do trato respiratório ${ }^{5}$. Apesar de não ser custo-efetivo, acreditamos que a radiografia de tórax "de base" pode ser útil e auxilliar na seleção da terapêutica, especialmente em pacientes selecionadas para tratamento domiciliar. Alguns autores observaram que a tomografia computadorizada (TC) de alta resolução pode revelar evidências de pneumonia em mais da metade das pacientes neutropênicas febris com radiografia de tórax norma ${ }^{20}$, mas o custo é bastante elevado e os benefícios restritos. Os métodos mais sensíveis, como a TC de alta resolução, devem ser indicados apenas em pacientes com febre persistente após as primeiras $48 \mathrm{~h}$ de antibiótico e radiografia de tórax normal'1.

\section{Conclusões}

Concluímos que não devemos realizar quimioterapia nas primeiras 24 horas após a cirurgia, especialmente em pacientes com idade superior a 60 anos, mas aguardar o restabelecimento das condições metabólicas após a reação de estresse secundário ao trauma cirúrgico. Também concluímos que devemos individualizar a realização concomitante de quimioterapia e radioterapia, podendo adiar esta última etapa até o término da quimioterapia, desde que não ultrapasse um intervalo de 20 a 24 semanas após a cirurgia.

\section{Conflito de interesse: não há.}

\section{SUMMARY}

\section{Febrile neutropenia in patients With BREAST CANCER SUBMITTED TO CHEMOTHERAPY: A 12 YEAR EXPERIENCE.}

PURPOSE. TO identify the characteristis of patients with breast cancer who developed febrile neutropenia and to establish risk factors for its incidence and parameters for an unfavorable evolution.

Patientsand Methods. A case-controlstudy was performed and included 65 patients: 13 patients presented febrile neutropenia and four controls per case matched by: date and number of previous chemotherapy cycles, drugs and doses used. The clinical and laboratory data were obtained from medical records. We utilized the odds ratio $(O R)$ and the $95 \%$ confidence interval (Cl) to estimate the significance of risk factors.

RESULTS. We identified two risk factors associated to occurrence offebrile neutropenia: use of chemotherapy within the first 24 hours post surgery (OR: 159.995\% Cl: 9.5 to 2699), and the concomitant use of chemotherapy and breast radiotherapy (OR: 108.3 95\% Cl: 4.9 to 2391). We did not observe a significant difference between cases and controls regarding age, body mass index, neutrophils and monocytes count prior to chemotherapy. Three patients died (23. 1\%), two of them were more than 60 years old, had no comorbidities, had received the first CMF within the first post surgery day and had surgical site infection.

CONCLUSIONS. The main risk factors associated with febrile neutropenia in patients with breast cancerwere: chemotherapy within the first 24 hours postsurgery, and concomitant chemotherapy and breast radiotherapy. As such, our study shows that these situations must be avoided. [Rev Assoc Med Bras 2004; 50(4): 363-6]

KEY WORDS: Febrile neutropenia, Breast cancer. Chemotherapy, Radiotherapy. 


\section{REFERÊNCIAS}

1. Valley AW. New treatment options for managing chemotherapy-induced neutropenia. Am J Health Syst Pharm 2002; 59:SII-S6.

2. Link BK, Budd GT, Scott S, Dickman E, Paul D, Lawless $G$, et al. Delivering adjuvant chemotherapy to women with early-stage breast carcinoma: current patterns of care. Cancer 2001:92:1354-67.

3. Choi CW, Sung HJ, Park KH, Yoon SY, Kim SJ, Oh SC, et al. Early lymphopenia as a risk factor for chemotherapy-induced febrile neutropenia. Am J Hematol 2003; 73:263-6.

4. Gandhi SK, Arguelles L, Boyer JG. Economic impact of neutropenia and febrile neutropenia in breast cancer: estimates from two national databases. Pharmacotherapy 200 I;2 I:684-90.

5. Hughes WT, Armstrong D, Bodey GP, Bow EJ, Brown AE, Calandra T, et al. 2002 Guidelines for the use of antimicrobial agents in neutropenic patients with cancer. Clin Infect Dis 2002; 34:730-5I.

6. Scott S. Identification of cancer patients at high risk of febrile neutropenia. Am J Health Syst Pharm 2002; 59:S16-S9.

7. Ozer H, Armitage JO, Bennett CL, Crawford J, Demetri GD, Pizzo PA, et al. 2000 update of recommendations for the use of hematopoietic colony-stimulating factors: evidencebased clinical practice guidelines. J Clin Oncol 2000; 18:3558-85.

8. International Union Against Cancer (UICC). TNM-Classification of malignant tumors. $5^{\text {th }}$ ed. New York: Wiley-Liss; 1997.
9. Barry MC, Condron CM, Watson RW, Redmond HP, El Jack M, Watson RG, et al. Pre-operative neutrophil and monocyte activation state predicts post-operative neutrophil and monocyte function. Eur J Surg 1997; 163:739-45.

10. Ciepichal J, Kubler A. Effect of general and regional anesthesia on some neutrophil functions. Arch Immunol Ther Exp 1998; 46: I83-92.

II. Faul C, Brufsky A, Gerszten K, Tlickinger J, Kunschner $A$, Jacob $H$, et al. Concurrent sequencing of full-dose CMF chemotherapy and radiation therapy in early breast cancer has no effect on treatment delivery. Eur I Cancer 2003; 39:763-8.

12. Isaac N, Panzarella T, Lau A, Mayers C, Kirbride P, Tannock IF, et al. Concurrent cyclophosphamide, methotrexate, and 5fluorouracil chemotherapy and radiotherapy for breast carcinoma: a well tolerated adjuvant regimen. Cancer 2002; 95:696-703.

13. Markiewicz DA, Schultz DJ, Haas JA, Harris EE, Fox KR, Glick JH, et al. The effects of sequence and type of chemotherapy and radiation therapy on cosmesis and complications after breast conservation therapy. Int J Radiat Oncol Biol Phys 1996; 35:66 I-8.

14. Dubey A, Recht A, Come SE, Gelman RS, Silver B, Harris JR, et al. Concurrent CMF and radiation therapy for early stage breast cancer: results of a pilot study. Int J Radiat Oncol Biol Phys 1999; 45:877-84.

15. Fiets WE, Van Helvoirt RP, Nortier JW, Van der Tweel I, Struikmans H. Acute toxicity of concurrent adjuvant radiotherapy and chemotherapy (CMF or $A C$ ) in breast cancer patients. a prospective, comparative, nonrandomised study. Eur J Cancer 2003; 39: $1081-8$

16. Levine JF, Coleman CN, Cox RS, Ray GR, Rogoway WM, Martinez A, et al. The effect of postoperative and primary radiation therapy on delivered dose of adjuvant cyclophosphamide, methotrexate, and 5-fluorouracil (CMF) chemotherapy in breast cancer. Cancer 1984; 53:237-41.

17. Banerjee TK, Hoehn JL, Greenlaw RH, Jacoby C. Chemotherapy drug dose alteration due to radiation therapy in an adjuvant situation in breast cancer. Am / Clin Oncol 1984; 7:437-42.

18. Ruo Redda MG, Verna R, Guarneri A, Sannazzari GL. Timing of radiotherapy in breast cancer conserving treatment. Cancer Treat Rev 2002; 28:5-10

19. Sickles EA, Greene WH, Wiernik PH. Clinical presentation of infection in granlocytopenic patients. Arch Intern Med 1975; 135:7 I 5-9.

20. Heussel CP, Kauczor HU, Heussel GE, Fischer $B$, Begrich M, Mildenberger $P$, et al. Pneumonia in febrile neutropenic patients and in bone marrow and blood stem cell transplant recipients: use of high resolution computed tomography. J Clin Oncol 1999; 17:796-805.

21 . Ninane V. Radiological and invasive diagnosis in the detection of pneumonia in febrile neutropenia. Int J Antimicrob Agents 2000; $|6: 9|-2$.

\footnotetext{
Artigo recebido: 13/1 |/03

Aceito para publicação: 26/02/04
} 